\title{
Pilot study of the relationship between psychotic manifestations and living environment
}

Antoine Longo, Hélène Charreire, Myriam Baron, Thierry Feuillet, Baptiste Pignon, Grégoire Baudin, Andrei Szoke and Franck Schurhoff

\section{(2) OpenEdition}

Journals

Electronic version

URL: https://journals.openedition.org/rfst/757

DOI: $10.4000 /$ rfst.757

ISSN: 2492-3672

This article is a translation of:

Étude pilote des relations entre manifestations psychotiques et environnement de résidence - URL https://journals.openedition.org/rfst/553 [fr]

Publisher

Espaces et SOciétés (UMR 6590)

Electronic reference

Antoine Longo, Hélène Charreire, Myriam Baron, Thierry Feuillet, Baptiste Pignon, Grégoire Baudin, Andrei Szoke and Franck Schurhoff, "Pilot study of the relationship between psychotic manifestations and living environment", Revue francophone sur la santé et les territoires [Online], Varia, Online since 17 October 2020, connection on 10 May 2021. URL: http://journals.openedition.org/rfst/757 ; DOI: https:// doi.org/10.4000/rfst.757

This text was automatically generated on 10 May 2021.

\section{cc) (1) (2)}

La Revue francophone sur la santé et les territoires est mise à disposition selon les termes de la Licence Creative Commons Attribution - Pas d'Utilisation Commerciale - Partage dans les Mêmes Conditions 4.0 International. 


\title{
Pilot study of the relationship between psychotic manifestations and living environment
}

\author{
Antoine Longo, Hélène Charreire, Myriam Baron, Thierry Feuillet, Baptiste \\ Pignon, Grégoire Baudin, Andrei Szoke and Franck Schurhoff
}

1 The study of subclinical psychotic manifestations (quantitative schizotypy) represents clinical and public health issues. These manifestations are more common than schizophrenia or depression, but rarely identified while it impacts the quality of life. Screening schizotypy in the general population would make it possible to propose prevention strategies. The objectives of the present research is to better understand the determinants and mechanisms of schizotypy in order to identify population at risk and hopefully be able to improve prevention and care. The hypothesis is that schizotypy is influenced both by individual and contextual characteristics (social, built).

2 Based on data collected during a survey in the city of Créteil, the aims of this article were to explore the relationships between representations (including size), socioeconomic and urban planning characteristics of the neighborhood of residence and schizotypy scores. In addition, a methodological contribution of this article is to measure and characterize self-defined neighborhoods.

\section{Methods}

3 Three administrative neighborhoods (IRIS) were selected in Créteil (France) according to socio-economic characteristics and geographical location related to the subway. Adult inhabitants ( $\geq 18$ years) within the 3 selected IRIS were invited to participate in a field survey in 2014. In the selected neighborhoods, 236 participants were recruited. The survey included questions on demographics and neighborhood perceptions, social relationships, health status as well as the personality aspects. In addition, participants were asked to draw what they considered as their residential neighborhood limits. A 
paper map of the city allowing respondents to draw the limits of their self-defined neighborhood was included in the survey. In the present study, we used data from participants who provided data on self-defined neighborhood limits $(n=225)$.

Data on built characteristics were defined using Geographical Information System (GIS) databases. The socio-economic context was characterized by the median level of income (INSEE census). Density of destinations, green space and a Land Use Mixed Index characterized the urban context. Schizotypy was assessed by self-report using the SPQ-B-Likert format questionnaire (Schizotypal Personality Questionnaire-BriefLikert format) based on 22 items (Ferchiou et al., 2017). This questionnaire includes cognitive-perceptual, interpersonal, and disorganized domains, which allowed to calculate 3 subscale schizotypy scores; a total score was also used. Associations between contextual characteristics and different schizotypy scores were performed with adjustment for gender and age.

\section{Results}

5 After adjustment, schizotypal scores are significantly and negatively associated with perceived neighborhood area except for the negative (social-interpersonal) sub-score. In addition, the (total) schizoptypal score is significantly higher in the group of subjects wishing to leave their neighborhood compared to groups of subjects who wish to stay in their neighborhood or who have no particular wish. Except for the negative subscore, subjects who report that their neighborhood of residence has a good reputation have significantly lower mean schizotypic scores than subjects who feel that the neighborhood has a bad reputation.

Interestingly, most associations between objective contextual variables (i.e. destination density, green space rate, median income level and land use mix index) and scores are not significant. The only exceptions are destination density (which is associated with total score and positive sub-score) and green spaces rate (associated with the negative sub-score). In other words, in our study population schizotypal scores are not (with a few exceptions) significantly associated with the characteristics of the built and social context of residence.

\section{Discussion and conclusion}

7 To your knowledge, this is the first study exploring the link between schizotypal scores and (objective, subjective) characteristics of the social and built residential context using self-defined neighborhood assessment.

8 First, all previous studies of the relationship between mental health and contextual characteristics were performed at the neighborhood level defined (only) by the administrative boundaries. Secondly, most of them focused on the relationships between incidence of clinically defined disorders (e.g. depression, anxiety, and schizophrenia) or depression and contextual characteristics such as deprivation, social isolation/fragmentation or urban level. For example, a recent research shown that individuals (living in Toronto) whose neighborhood representations are negative in terms of social environment / reputation and built environment also reported higher scores of depression and anxiety than those with positive representation (Olsen et al., 
2017). In addition, a review of the literature (Gong et al., 2016) suggests that elements of architecture, density of green space, mix land use, industrial activity and density of traffic would have an impact on depression and anxiety.

9 In our study population, schizotypal scores are lower in groups of subjects who report large perceived neighborhood or who have a positive representation of their neighborhood of residence. However, as our study has some limitations, caution is needed when interpreting and generalizing the results. Due to its cross-sectional nature, temporal dimension of relation as well as impact of residential neighborhood across life cannot be assessed.

It would be necessary to develop analyzes on a larger sample based on cohort design between different cities/neighborhoods to take into account life course of individuals.

Scores of schizotypy by size of self-defined residential neighborhood (tercile) after adjustment on age and gender $(n=225)$

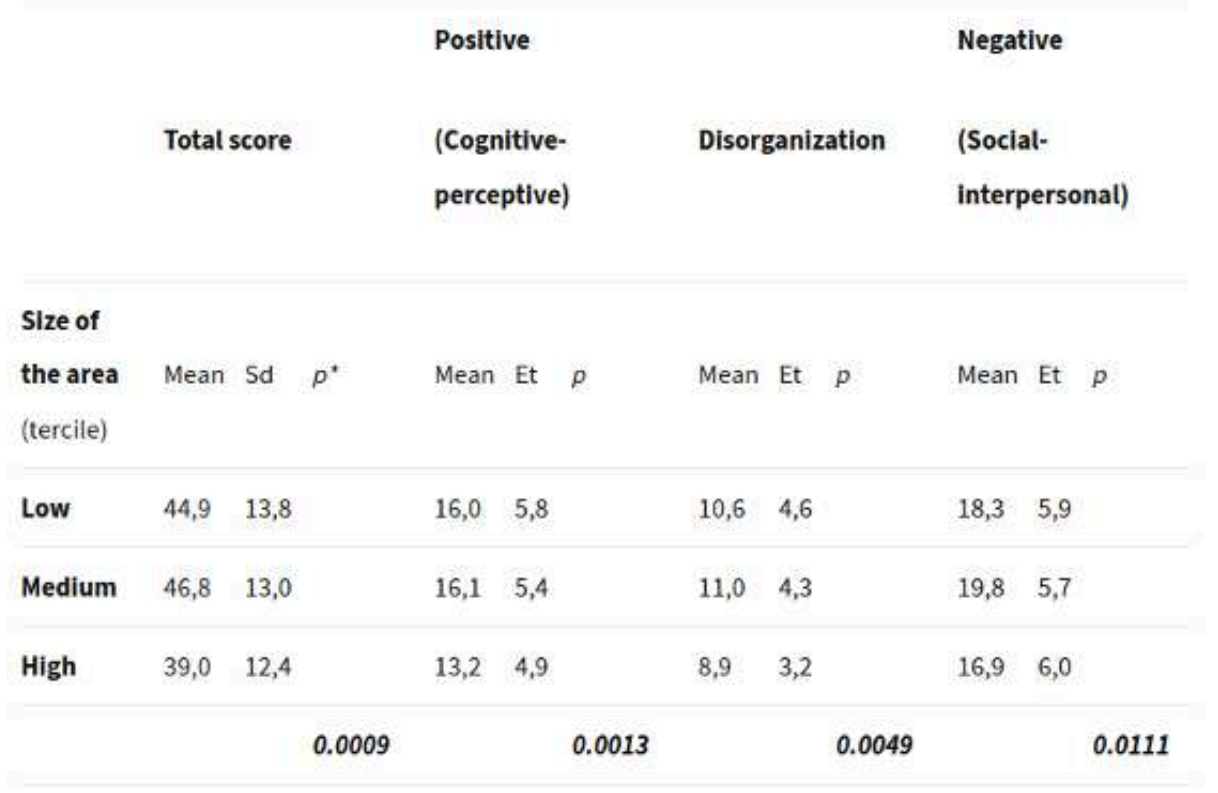

Sd: Standard deviation

`Between groups (tercile)

\section{BIBLIOGRAPHY}

Ferchiou, A., Todorov, L., Lajnef, M., Baudin, G., Pignon, B., Richard, J.-R., ... Schürhoff, F. (2017). Analyse de la structure factorielle de la version brève du questionnaire de personnalité schizotypique (SPQ-B) - format Likert - en population générale en France. L'Encéphale, 43(6), 558563. DOI:10.1016/j.encep.2016.05.011 
Gong, Y., Palmer, S., Gallacher, J., Marsden, T., \& Fone, D. (2016). A systematic review of the relationship between objective measurements of the urban environment and psychological distress. Environment International, 96, 48-57. DOI:10.1016/j.envint.2016.08.019

Olsen, J., Dundas, R., \& Ellaway, A. (2017). Are Changes in Neighbourhood Perceptions Associated with Changes in Self-Rated Mental Health in Adults? A 13-Year Repeat Cross-Sectional Study, UK. International Journal of Environmental Research and Public Health, 14(12), 1473. DOI:10.3390/

ijerph14121473

\section{INDEX}

Keywords: psychotic disorders, schizotypy, schizophrenia, quality of life, residence, places of life Geographical index: Île-de-France, Créteil

\section{AUTHORS}

\section{ANTOINE LONGO}

Université Paris Est Créteil, LAB’URBA, F-94010 Créteil, France

\section{HÉLÈNE CHARREIRE}

Université Paris Est Créteil, LAB’URBA, F-94010 Créteil, France

\section{MYRIAM BARON}

Université Paris Est Créteil, LAB’URBA, F-94010 Créteil, France

\section{THIERRY FEUILLET}

Université Paris 8, LADYSS, UMR 7533 CNRS, France

\section{BAPTISTE PIGNON}

AP-HP, Hôpital Henri Mondor, Département Medico-Universitaire de Psychiatrie et d'Addictologie (DMU IMPACT), Fédération Hospitalo-Universitaire de Médecine de Précision (FHU ADAPT) F-94010, France ; INSERM U955, IMRB, Laboratoire Neuro-psychiatrie translationnelle, F-94010 Creteil, France; Fondation FondaMental, Créteil, 94000, France

\section{GRÉGOIRE BAUDIN}

AP-HP, Hôpital Henri Mondor, Département Medico-Universitaire de Psychiatrie et d'Addictologie (DMU IMPACT), Fédération Hospitalo-Universitaire de Médecine de Précision (FHU ADAPT) F-94010, France ; INSERM U955, IMRB, Laboratoire Neuro-psychiatrie translationnelle, F-94010 Creteil, France; Fondation FondaMental, Créteil, 94000, France

\section{ANDREI SZOKE}

AP-HP, Hôpital Henri Mondor, Département Medico-Universitaire de Psychiatrie et d'Addictologie (DMU IMPACT), Fédération Hospitalo-Universitaire de Médecine de Précision (FHU ADAPT) F-94010, France ; INSERM U955, IMRB, Laboratoire Neuro-psychiatrie translationnelle, F-94010 Creteil, France; Fondation FondaMental, Créteil, 94000, France 


\section{FRANCK SCHURHOFF}

AP-HP, Hôpital Henri Mondor, Département Medico-Universitaire de Psychiatrie et d'Addictologie (DMU IMPACT), Fédération Hospitalo-Universitaire de Médecine de Précision (FHU ADAPT) F-94010, France ; INSERM U955, IMRB, Laboratoire Neuro-psychiatrie translationnelle, F-94010 Creteil, France ; Université Paris- Est Créteil (UPEC), Créteil, 94000, France ; Fondation FondaMental, Créteil, 94000, France 